\title{
Antibody Response of Monkeys to Invasion Plasmid Antigen D after Infection with Shigella spp.
}

\author{
EDWIN V. OAKS, ${ }^{1 *}$ WILLIAM D. PICKING, ${ }^{2}$ AND WENDY L. PICKING ${ }^{2}$ \\ Department of Enteric Infections, Walter Reed Army Institute of Research, Washington, D.C. 20307, ${ }^{1}$ \\ and Department of Biology, St. Louis University, St. Louis, Missouri $63103^{2}$
}

Received 3 November 1995/Returned for modification 15 December 1995/Accepted 18 January 1996

\begin{abstract}
The antigen preparation most often used for determining the levels of antibodies to virulence-associated proteins of Shigella spp. consists of a mixture of proteins (including IpaB, IpaC, IpaD, and VirG*) extracted from virulent shigellae with water (water extract). To overcome the lack of specificity for individual antigens in the water-extract enzyme-linked immunosorbent assay (ELISA), the ipaD gene from $S$. flexneri has been cloned, expressed to a high level, and purified for use in a new ELISA for the determination of the levels of antibody against IpaD in monkeys and humans challenged with shigellae. The IpaD ELISA for serum immunoglobulins G and A correlated well with the water-extract ELISA in that monkeys infected with $S$. flexneri or $S$. sonnei responded with high serum antibody titers in both assays. The IpaD assay required less antigen per well, had much lower background levels, and did not require correction with antigens from an avirulent organism. In conjunction with the water-extract ELISA, it was possible to identify infected animals that did not respond to IpaD but did produce antibodies that reacted in the water-extract ELISA. This indicates that even though IpaB, IpaC, and IpaD are essential for the invasiveness phenotype, the infected host does not always produce antibodies against all components of the invasiveness apparatus.
\end{abstract}

Bacillary dysentery is a severe diarrheal disease caused by Shigella species. Several essential virulence factors of Shigella spp., including the invasion plasmid antigen (Ipa) proteins (IpaB, IpaC, and IpaD), VirG (IcsA), and lipopolysaccharide (LPS) elicit substantial antibody responses in the serum as well as in secretions after infection $(1,5,6,10,11,13)$. The most frequently used serological assays for measuring the antibody response against Shigella protein antigens are Western blots (immunoblots) and enzyme-linked immunosorbent assays (ELISAs). Western blots provide qualitative and semiquantitative information about antibodies against specific protein antigens, but they must be interpreted carefully. For example, in the IpaB region of blots, three serologically recognized antigens, including IpaB, IpaH, and HSP60, exist. The titers of antibodies are more readily determined by ELISAs. Two antigens routinely used in ELISAs for Shigella serodiagnosis are purified LPS (which must be prepared for each Shigella serotype to be analyzed) and the water-extractable antigens (1, 5, $10,13)$. The latter antigen preparation is a heterogenous product which includes both invasiveness plasmid antigens (IpaB, IpaC, IpaD, and VirG*) and chromosomally encoded proteins and $\operatorname{LPS}(9,10,15)$. This antigen preparation is frequently used for measuring antibody levels in serum and secretions as well as for the detection of circulating antibody-secreting cells producing antibodies against the virulence-specific antigen complex $(3,4)$. It can be used for any species of Shigella or enteroinvasive Escherichia coli because the ipa genes are conserved in all serotypes. Unfortunately, it does not measure antibody levels against specific Ipa proteins or VirG, and it requires a correction for antibodies against chromosomally encoded proteins. This is accomplished by preparing a water extract from a plasmid-free Shigella strain that is the same serotype as the virulent preparation. To overcome the shortcomings of the water-extract ELISA, we have developed a new ELISA using purified, recombinant $\mathrm{IpaD}$ protein. IpaD is present in the

* Corresponding author. Phone: (202) 782-3010. Fax: (202) 782-0748. water extract from virulent organisms and is a crucial factor in the invasiveness of shigellae $(7,9,10)$. It is located in the outer membrane of shigellae, is exposed on the surface, and is possibly involved in the modulation of secretion of IpaB and IpaC $(7,9,10)$. The IpaD ELISA, described here, will provide specificity and sensitivity in serological assays for monitoring the immune response to virulence-associated antigens after infection with shigellae. This will be useful in determining infection with Shigella spp. in nonimmune humans and higher primates in outbreaks of diarrheal disease as well as in seroprevalence surveys.

The PCR was used to produce a 1,000-bp ipaD fragment for insertion into the plasmid expression vector $\mathrm{pET}-15 \mathrm{~b}$. Primers, based on the published nucleotide sequence of ipaD (14), were designed to give an ipaD-containing DNA fragment that could be inserted in frame into pET-15b after each was NdeI-BamHI digested. The resulting PCR product was treated with NdeI and BamHI and ligated into NdeI-BamHI-digested pET-15b. E. coli BL21(DE3)pLysS was transformed with the ligation mixture, and transformants were selected on Luria-Bertani agar containing $0.03 \mathrm{mg}$ of ampicillin per ml. Transformants were screened for inserts by the appearance of a 1,000-bp PCR product by using the T7 RNA polymerase promoter and terminator sequences as primers. Insertion of the ipaD fragment was confirmed by double-stranded DNA sequencing.

Overexpression of the resulting ipaD fusion gene in $E$. coll BL21 (DE3)pLysS was possible with IPTG (isopropyl- $\beta$-Dthiogalactopyranoside) induction (Fig. 1). The fusion protein product with a size of about $42 \mathrm{kDa}$ (Fig. 1) consisted of full-length IpaD fused to a 3-kDa leader peptide containing six histidine residues. This leader peptide enabled the fusion protein to be affinity purified by passing a cytosolic extract, containing the recombinant protein, over a $\mathrm{Ni}^{2+}$ column followed by elution of the IpaD fusion protein with $1 \mathrm{M}$ imidazole. The IpaD fusion protein was approximately $95 \%$ pure as determined with Coomassie-stained gels, and it reacted with three different rabbit anti-IpaD serum samples made against three different IpaD peptides (9). 


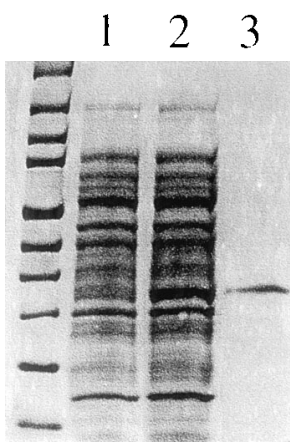

FIG. 1. Affinity purification of the IpaD-fusion protein. E. coli BL21(DE3)pLysS harboring the recombinant plasmid pWPD10 was induced with IPTG, resulting in the appearance of a protein observed on Coomassiestained sodium dodecyl sulfate-10\% polyacrylamide gels with an apparent molecular mass of $42 \mathrm{kDa}$ (lane 2). No such protein species was observed in uninduced cells (lane 1). The crude cytosolic extract was passed over an immobilized support to which $\mathrm{Ni}^{2+}$ had been reversibly chelated. The column was washed extensively, and the IpaD fusion protein eluted with the same buffer containing $1 \mathrm{M}$ imidazole. The purified IpaD fusion protein (lane 3), prepared in this way, was routinely greater than $95 \%$ pure as judged with Coomassie-stained sodium dodecyl sulfate-polyacrylamide gels. Molecular mass standards (in the unmarked lane) are $212,158,116,97,66,56,43,37,26$, and $20 \mathrm{kDa}$, respectively, from top to bottom.

To date it has not been possible to measure antibody levels against individual Ipa proteins in an ELISA because of the lack of pure, stable Ipa proteins. To establish the usefulness of the IpaD ELISA, we compared the serum antibody levels against IpaD, water-extracted antigens, and LPS in monkeys infected with $S$. flexneri 2 a.

Purified IpaD, diluted in phosphate-buffered saline (PBS; pH 7.4), was used to coat polystyrene 96-well plates (Corning/ Costar, Cambridge, Mass.) at a final concentration of $0.05 \mu \mathrm{g}$ per well. The antigen was incubated on the plates overnight at $4^{\circ} \mathrm{C}$. The wells were subsequently blocked with $2 \%$ casein in a Tris-buffered saline solution. Immune sera, diluted in casein, were incubated with the antigen for $2 \mathrm{~h}$. After the wells were washed with PBS containing $0.05 \%$ Tween 20 , alkaline phosphatase-conjugated anti-human immunoglobulin $\mathrm{G}$ (IgG) or IgA (Kirkegaard \& Perry, Gaithersburg, Md.) was added for 1 $\mathrm{h}$, the wells were washed, and finally, the substrate (paranitrophenylphosphate, $1 \mathrm{mg} / \mathrm{ml}$ in diethanolamine buffer) was added to each well and allowed to develop for $30 \mathrm{~min}$. All incubations with antisera and washes were performed at room temperature. The optical density (OD) was determined with a

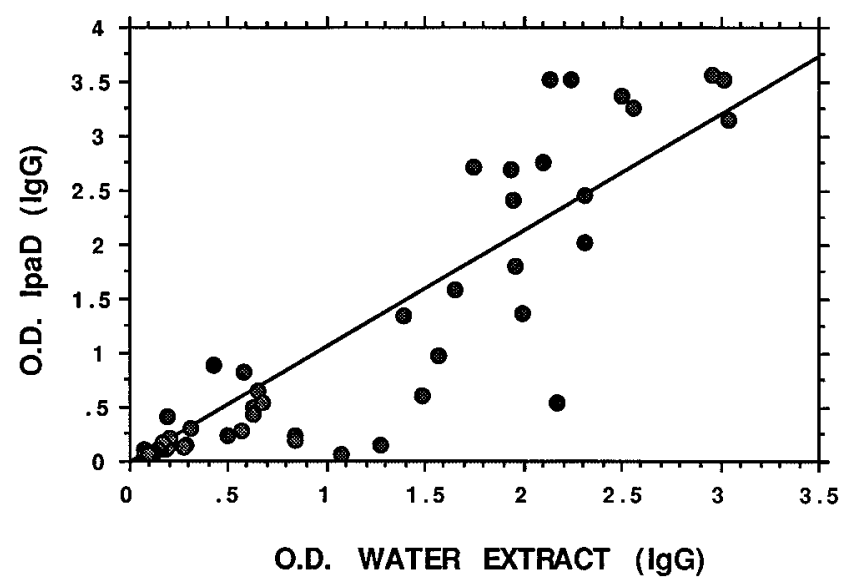

FIG. 2. Correlation between the IpaD ELISA and the water extract ELISA. A total of 47 serum samples were collected from monkeys 2 weeks after oral challenge with $S$. flexneri 2 a. All serum samples were diluted $1 / 800$ for this analysis and were probed with an anti-IgG conjugate. A comparison of the optical density values obtained for the IpaD ELISA versus those for the waterextract ELISA is shown ( $r$ of $0.942, P$ of $<0.0001$ ). The solid line represents the best fit obtained by regression analysis.

Molecular Dynamics microplate reader at a wavelength of 405 $\mathrm{nm}$. The levels of serum antibodies against LPS and virulenceassociated antigens extracted with water were measured with an ELISA, as previously described (10).

Antibody levels to IpaD, water extract, and LPS were determined for sera collected from 47 monkeys from four different treatment groups, 2 weeks after infection with $S$. flexneri 2 a (Table 1). The quantity of shigellae administered orally ranged from $2 \times 10^{3} \mathrm{CFU}$ per monkey to $2 \times 10^{9} \mathrm{CFU}$ per monkey, with each successive group of 12 monkeys receiving 100 -foldmore shigellae. The various doses of shigellae gave a range of immune responses and clinical disease. The baseline anti-IpaD levels were uniformly low for all monkeys. Infection with lower doses of shigellae $\left(2 \times 10^{3}\right.$ and $\left.2 \times 10^{5} \mathrm{CFU}\right)$ did not produce disease and resulted in a minimal serum antibody response. At the highest dose $\left(2 \times 10^{9} \mathrm{CFU}\right.$ per monkey), 10 of 11 monkeys had diarrhea, 10 of 11 produced serum IgG antibodies against IpaD, and all 11 were positive for IgG antibodies against the water extract (Table 1). Similar responses for IgA against IpaD and $\mathrm{IgG}$ for LPS were found. The level of response against LPS appeared somewhat lower in the group of animals receiving 2 $\times 10^{7}$ CFU. The data in Fig. 2 indicate that a correlation

TABLE 1. Serological response to IpaD, water extract, and LPS in monkeys challenged with various doses of $S$. flexneri $2 \mathrm{a}$

\begin{tabular}{|c|c|c|c|c|c|c|c|c|c|}
\hline \multirow{3}{*}{ Dose (CFU) } & \multirow{3}{*}{$\begin{array}{l}\text { No. of animals infected/ } \\
\text { no. tested }^{a}\end{array}$} & \multicolumn{4}{|c|}{ Mean $\mathrm{OD} \pm \mathrm{SD}^{b}$} & \multicolumn{4}{|c|}{$\begin{array}{l}\text { No. of animals that seroconverted against antigen } \\
\text { indicated/no. tested }\end{array}$} \\
\hline & & \multicolumn{2}{|c|}{$\mathrm{IpaD} / \mathrm{IgG}$} & \multicolumn{2}{|c|}{$\mathrm{IpaD} / \mathrm{IgA}$} & \multirow{2}{*}{$\operatorname{IpaD}(\operatorname{IgG})^{\mathrm{d}}$} & \multirow{2}{*}{$\operatorname{IpaD}(\operatorname{Ig} \mathrm{A})^{\mathrm{d}}$} & \multirow{2}{*}{ Water extract } & \multirow{2}{*}{$\mathrm{LPS}^{d}$} \\
\hline & & Preinfection & Postinfection & Preinfection & Postinfection & & & & \\
\hline $2.0 \times 10^{3}$ & $0 / 12$ & $0.26 \pm 0.21$ & $0.23 \pm 0.17$ & $0.44 \pm 0.67$ & $0.37 \pm 0.60$ & $0 / 12$ & $0 / 12$ & $0 / 12$ & $0 / 12$ \\
\hline $2.0 \times 10^{5}$ & $0 / 12$ & $0.33 \pm 0.48$ & $0.34 \pm 0.37$ & $0.46 \pm 1.01$ & $0.49 \pm 0.97$ & $1 / 12$ & $1 / 12$ & $2 / 12$ & $0 / 12$ \\
\hline $2.0 \times 10^{7}$ & $2 / 12$ & $0.62 \pm 0.73$ & $1.67 \pm 1.01^{d}$ & $0.25 \pm 0.18$ & $0.82 \pm 0.63^{d}$ & $8 / 12$ & $8 / 12$ & $7 / 12$ & $4 / 12$ \\
\hline $2.0 \times 10^{9}$ & $10 / 11$ & $0.32 \pm 0.18$ & $2.52 \pm 1.27^{d}$ & $0.19 \pm 0.19$ & $0.93 \pm 0.80^{d}$ & $10 / 11$ & $8 / 11$ & $11 / 11$ & $10 / 11$ \\
\hline
\end{tabular}

${ }^{a}$ Monkeys exhibiting diarrhea within $72 \mathrm{~h}$ of infection were considered positive for disease (infected) due to the Shigella challenge.

${ }^{b}$ The mean OD values at a 1/1,000 dilution of the preinfection and postinfection serum samples are given. The postinfection serum samples were collected 14 days after challenge.

${ }^{c}$ The various antigens used to determine the serological response included purified recombinant IpaD, water-extractable virulence-associated antigens (water extract), and LPS from S. flexneri 2a. IgA and IgG levels were determined against IpaD. Only the IgG level was determined against water extract and LPS. Positive samples represent those serum samples which had a twofold-higher OD value in the postinfection serum sample than in the preinfection serum sample.

${ }^{d}$ The difference between the mean OD values was statistically significant, as determined by the Wilcoxon signed rank test $(P<0.005)$. 
TABLE 2. Serum IgG response to IpaD in monkeys challenged with either $S$. flexneri 2 a or $S$. sonnei

\begin{tabular}{|c|c|c|c|c|}
\hline \multirow{2}{*}{$\begin{array}{l}\text { Challenge } \\
\text { species }^{a}\end{array}$} & \multirow{2}{*}{$\begin{array}{l}\text { No. of animals } \\
\text { with clinical } \\
\text { signs of disease/ } \\
\text { no. tested }\end{array}$} & \multicolumn{2}{|c|}{ Mean OD $\pm \mathrm{SD}^{b}$} & \multirow{2}{*}{$\begin{array}{c}\text { No. of } \\
\text { monkeys } \\
\text { with IpaD } \\
\text { sero- } \\
\text { conversion }\end{array}$} \\
\hline & & $\begin{array}{l}\text { Preinfec- } \\
\text { tion }\end{array}$ & $\begin{array}{l}\text { Postinfec- } \\
\text { tion }^{d}\end{array}$ & \\
\hline S. flexneri $2 \mathrm{a}$ & $8 / 12$ & $0.06 \pm 0.03$ & $1.10 \pm 0.77$ & 10 \\
\hline S. sonnei & $7 / 12$ & $0.19 \pm 0.18$ & $1.01 \pm 0.62$ & 12 \\
\hline
\end{tabular}

${ }^{a}$ Monkeys were challenged orally with $2 \times 10^{10} \mathrm{CFU}$ of either $S$. flexneri $2 \mathrm{a}$ or $S$. sonnei. The details of this challenge study have been previously published (2).

${ }^{b}$ The mean OD values at a 1/1,000 dilution of the pre- and postinfection samples are given. The postinfection serum samples were collected 9 days after challenge.

${ }^{c}$ Those animals which had a twofold-higher OD value (at a 1/1,000 dilution) in the postinfection serum sample than in the preinfection sample were considered positive (to have seroconverted).

${ }^{d}$ The difference between the pre- and postinfection mean OD value had a $P$ value of $<0.005$ by the Wilcoxon signed rank test.

between the IpaD and water-extract ELISA ( $r$ of $0.942, P$ of $<0.0001)$ existed when IgG levels were determined. Even though the relationship between the IpaD (IgG) ELISA and the water-extract (IgG) ELISA was very strong, there were two monkeys (of 47) that had serum IgG antibodies to the waterextracted antigens but not to IpaD. Sera from these monkeys did not react with IpaD on Western blots but did react with IpaB and IpaC (data not shown). Regression analysis between the LPS and IpaD ELISA and the LPS and water-extract ELISA gave $r$ values of $0.814(P$ of $<0.0001)$ and 0.809 ( $P$ of $<0.0001)$, respectively.

The water-extract ELISA is useful in determining antibody responses against all members of the genus Shigella in large part because the ipa genes, which encode the dominant antigens in the water extract, are conserved in all invasive Shigella spp. and enteroinvasive E. coli. The data in Table 2 indicate that, as expected, monkeys infected with either $S$. sonnei or $S$. flexneri produced serum antibodies against IpaD. Interestingly, 2 of the $12 \mathrm{~S}$. flexneri-infected monkeys did not show increased levels of $\mathrm{IgG}$ against IpaD in serum collected 9 days postinfection. Similar to the single animal in the group inoculated with $2 \times 10^{9} \mathrm{CFU}$, these two animals did produce antibodies against the water extract, and specifically against IpaB and IpaC (as determined by Western blot analysis) (9).

In this study, we demonstrated that purified, recombinant IpaD can be used to monitor the specific immune response in monkeys infected with a Shigella sp. The assay requires small quantities of antigen, has low background levels, and is specific for IpaD. Unlike the water-extract ELISA, it does not require correction with an antigen from an avirulent source because it uses a pure antigen. In addition, the recombinant $\mathrm{IpaD}$ is expressed to high levels in the pET15b vector, is relatively stable (compared with the easily degraded IpaB and IpaC), and is easy to purify via affinity chromatography on a nickel column.

The IpaD ELISA correlated very well with the water-extract ELISA, which was expected since IpaD is present in the water extract. It was noted that a few monkeys infected with shigellae did not produce detectable antibodies in the IpaD ELISA but were positive in the water-extract ELISA. These serum samples, which were all evaluated by Western blot, were negative for IpaD and positive for IpaB and IpaC antibodies (9). The ability of the water-extract ELISA to pick up more positives than the IpaD ELISA is very likely related to a higher frequency of antibody responses against IpaB and IpaC in infected animals. Therefore, it is unlikely that the IpaD ELISA can replace the water-extract ELISA but that it will be useful because of its specificity for IpaD and the lack of background problems. Sensitivity approaching that of the water-extract ELISA will likely occur when purified IpaB or IpaC becomes available. Even so, by using the water-extract ELISA along with the IpaD ELISA, it is possible to identify subgroups of infected individuals that have unique immunological responses to the Ipa proteins. The ability to distinguish subgroups of infected individuals will enable a better understanding of the host immune response to Shigella infection. In addition, these results indicate that all components of the invasiveness protein complex of Shigella spp. $(7,8)$ are not recognized equally by all infected animals.

The IpaD ELISA may also be useful for seroepidemiological studies designed to identify populations with a high or low frequency of exposure to Shigella spp. without the need to consider multiple serotypes of the genus Shigella. For example, in a group of healthy, adult U.S. citizens, the incidence of individuals with IpaD antibodies was approximately $15 \%$ (9). A similar frequency was noted for antibodies against the water extract. In areas of endemicity, the frequency of IpaD antibodies in an adult population would probably be much higher (11, $13)$.

As other plasmid-encoded antigens, such as IpaB, IpaC, and VirG, are purified, it will be possible to evaluate the specific immune response both at the humoral and the totally uncharacterized cellular level for each of these components. This type of information, in conjunction with more-detailed analysis at the peptide level (12), may be necessary for determining immune response patterns that are correlated with disease outcome or protection against future disease.

We thank L. Van de Verg and K. R. Turbyfill for helpful comments and suggestions concerning the manuscript and F. Puente for technical assistance.

\section{REFERENCES}

1. Dinari, G., T. L. Hale, S. W. Austin, and S. B. Formal. 1987. Local and systemic antibody responses to Shigella infection in Rhesus monkeys. J. Infect. Dis. 155:1065-1069.

2. Formal, S. B., E. V. Oaks, R. E. Olsen, M. Wingfield-Eggleston, P. J. Snoy, and J. P. Cogan. 1991. Effect of prior infection with virulent Shigella flexneri 2a on the resistance of monkeys to subsequent infection with Shigella sonnei. J. Infect. Dis. 164:533-537.

3. Kotloff, K. L., D. A. Herrington, T. L Hale, J. W. Newland, L. Van De Verg, J. P. Cogan, P. J. Snoy, J. C. Sadoff, S. B. Formal, and M. M. Levine. 1992. Safety, immunogenicity, and efficacy in monkeys and humans of invasive Escherichia coli K-12 hybrid vaccine candidate expressing Shigella flexneri 2a somatic antigen. Infect. Immun. 60:2218-2224.

4. Li, A., A. Karnell, P. T. Huan, P. D. Cam, N. B. Minh, L. N. Tram, N. P. Quy, D. D. Trach, K. Karlsson, G. Lindberg, and A. A. Lindberg. 1993. Safety and immunogenicity of the live oral auxotrophic Shigella flexneri SFL124 in adult Vietnamese volunteers. Vaccine 11:180-189.

5. Li, A., Z. C. Rong, E. Ekwall, U. Forsum, and A. A. Lindberg. 1993. Serum antibody responses against Shigella lipopolysaccharides and invasion plasmid-coded antigens in Shigella-infected Swedish patients. Scand. J. Infect. Dis. 25:569-577.

6. Li, A., C. R. Zhao, E. Ekwall, and A. A. Lindberg. 1994. Serum IgG antibody responses to Shigella invasion plasmid-coded antigens detected by immunoblot. Scand. J. Infect. Dis. 26:435-445.

7. Menard, R., P. Sansonetti, and C. Parsot. 1994. The secretion of the Shigella flexneri Ipa invasins is activated by epithelial cells and controlled by IpaB and IpaD. EMBO J. 13:5293-5302.

8. Menard, R., P. Sansonetti, C. Parsot, and T. Vasselon. 1994. Extracellular association and cytoplasmic partitioning of the IpaB and IpaC invasins of Shigella flexneri. Cell 79:515-525.

9. Oaks, E. V. Unpublished observation.

10. Oaks, E. V., T. L. Hale, and S. B. Formal. 1986. Serum immune response to Shigella protein antigens in Rhesus monkeys and humans infected with Shigella spp. Infect. Immun. 53:57-63.

11. Oberhelman, R. A., D. J. Kopecko, E. Salazar-Lindo, E. Gotuzzo, J. M. Buysse, M. M Venkatesan, A. Yi, C. Fernandez-Prada, M. Guzman, R. Leon-Barua, and R. B. Sack. 1991. Prospective study of systemic and mu- 
cosal immune responses in dysenteric patients to specific Shigella invasion plasmid antigens and lipopolysaccharides. Infect. Immun. 59:2341-2350.

12. Turbyfill, K. R., S. W. Joseph, and E. V. Oaks. 1995. Recognition of three epitopic regions on invasion plasmid antigen $\mathrm{C}$ (IpaC) by immune sera of Rhesus monkeys infected with Shigella flexneri 2a. Infect. Immun. 63:39273935.

13. Van De Verg, L. L., D. A. Herrington, J. Boslego, A. A. Lindberg, and M. M. Levine. 1992. Age-specific prevalence of serum antibodies to the invasion plasmid and lipopolysaccharide antigens of Shigella species in Chilean and North American populations. J. Infect. Dis. 166:158-161.

14. Venkatesan, M. M., J. M. Buysse, and D. J. Kopecko. 1988. Characterization of invasion plasmid antigen genes (ipaBCD) from Shigella flexneri. Proc. Natl. Acad. Sci. USA 85:9317-9321.

15. Venkatesan, M. M., J. M. Buysse, and E. V. Oaks. 1992. Surface presentation of Shigella flexneri invasion plasmid antigens requires the products of the spa locus. J. Bacteriol. 174:1990-2001. 